PAPER • OPEN ACCESS

Phase interference for probing topological fractional charge in a $\mathrm{BiSbTeSe}_{2}$-based Josephson junction array

To cite this article: J M Brevoord et al 2021 Nanotechnology 32435001

View the article online for updates and enhancements.

The Electrochemical Society

Advancing solid state \& electrochemical science \& technolog 2021 Virtual Education

\title{
Intensive Short Courses
}

Sun, Oct 10 \& Mon, Oct 11

Providing students and professionals with in-depth education on a wide range of topics

Early registration deadline: Sep 13, 2021 


\title{
Phase interference for probing topological fractional charge in a $\mathrm{BiSbTeSe}_{2}$-based Josephson junction array
}

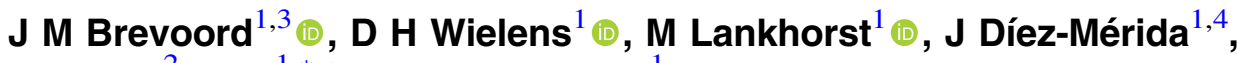

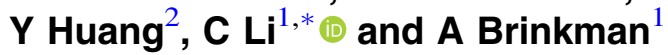 \\ ${ }^{1} \mathrm{MESA}^{+}$Institute for Nanotechnology, University of Twente, The Netherlands \\ ${ }^{2}$ Van der Waals-Zeeman Institute, IoP, University of Amsterdam, The Netherlands \\ E-mail: chuan.li@utwente.nl
}

Received 18 May 2021, revised 5 July 2021

Accepted for publication 14 July 2021

Published 2 August 2021

\begin{abstract}
Fractional charges can be induced by magnetic fluxes at the interface between a topological insulator (TI) and a type-II superconductor due to axion electrodynamics. In a Josephson junction array with a hole in the middle, these electronic states can have phase interference in an applied magnetic field with $4 \times 2 \pi$ period, in addition to the $2 \pi$ interference of the Cooper pairs. Here, we test an experimental configuration for probing the fractional charge and report the observation of phase interference effect in superconducting arrays with a hole in the middle in both Au- and TI-based devices. Our numerical simulations based on resistive shunted capacitive junction model are in good agreement with the experimental results. However, no clear sign of an axion charge-related interference effect was observed. We will discuss possible reasons and perspectives for future experiments.
\end{abstract}

Supplementary material for this article is available online

Keywords: topological insulator, fractional charge, Josephson junction arrays, phase interference, topological superconductivity

(Some figures may appear in colour only in the online journal)

\section{Introduction}

The study of topological insulators (TIs) has led to the discovery of a class of materials which are characterized by conducting surface states and an insulating bulk [1-4]. Combining the unique properties of topological materials

${ }^{3}$ Present address: QuTech, Delft University of Technology, The Netherlands.

${ }^{4}$ Present address: ICFO-Institut de Ciències Fotòniques, The Barcelona Institute of Science and Technology, Spain.

* Author to whom any correspondence should be addressed. with superconductors can lead to gapless bound states, which in turn could lead to the observation of Majorana fermions [5]. While signatures of these Majorona fermions have been observed in experiments [6-8], definitive proof is still absent. More recently, it has been shown theoretically that vortices in a type-II superconductor placed on top of a TI can bind fractional charges of $e / 4$ at the interface due to a manifestation of axion electrodynamics [9]. The concept of axion electrodynamics, first studied in high-energy physics, was later adopted in the framework of topological materials [10]. At the interfaces of a TI, the Maxwell's equations are modified by an $\mathbf{E} \cdot \mathbf{B}$-term which is non-vanishing due to the change of the so-called axion angle $\theta$ which is $\pi$ for a TI and is zero for trivial materials. The unconventional coupling between the electric and magnetic field can give rise to exotic effects, such as magnetic monopoles [11], the chiral anomaly [12-14] and the Witten effect [15]. Localized fractional 
charges can also be found in spatial-symmetry protected materials, namely topological crystalline insulators. Recently, this has been experimentally realized in metamaterials [16-18]. Here, we follow the proposal of Nogueira et al [9] to place a Josephson junction array (JJA) on top of a TI to measure the interference of fractional charges that are generated due to the Witten effect acting on vortices in the type-II superconductor. Furthermore, it was shown that the fractional charge leads to fractional angular momentum. The predicted fractional angular momentum of the magnetic vortices at the $\mathrm{TI}$ interface are of special interest as interchanging of these vortices leads to a change of phase of the wavefunction that is between zero and $\pi$. The manipulation of these anyons, with their non-Abelian statistics, is the key of topological quantum computing.

Axion electrodynamics captures the coupling of the electric and magnetic fields entering or leaving TIs in the axion Lagrangian density [15], $\mathcal{L}_{\theta}=2 \alpha \sqrt{\epsilon_{0} / \mu_{0}}(\theta / 2 \pi) \mathbf{E} \cdot \mathbf{B}$, where $\mathbf{E}$ and $\mathbf{B}$ are the electric and magnetic field respectively, $\theta$ is the $2 \pi$-periodic axion angle and $\alpha$ the fine-structure constant. As a result of the coupling of the electric and magnetic field, field theory shows that the differential form of the Gauss's law becomes $\nabla \cdot \mathbf{E}+\kappa c \nabla \theta \cdot \mathbf{B}=\rho / \epsilon_{0}$, where $\kappa=1 /\left(4 \pi^{2} \epsilon_{0}\right) \cdot e^{2} / \hbar c$. In the absence of external charges, integrating over the interface of a TI $(\theta=\pi)$ and a trivial insulator $(\theta=0)$, results in

$$
\frac{Q}{\epsilon_{0}}=-\kappa c \theta \int B(r) \mathrm{d}^{2} r .
$$

In the presence of quantized flux, such as vortices in a type-II superconductors, the charge at the surface of a TI is fractional. One flux quantum, $\phi_{0}$, will attain a fractional charge of $Q=\frac{e}{4}$. Fractional charge at a TI surface is proposed to be measured by an interference experiment in a JJA [9]. In the presence of an external magnetic field, a Josephson vortex is formed when there is a phase difference of $2 \pi$ across a closed loop in a JJA. If the critical current, $I_{c}$, of the Josephson Junctions of the closed loop is high enough, Josephson vortices have a flux equal to a single flux quantum, $\phi_{0}$. As a result of equation (1), each Josephson vortex at the a TI surface in a JJA will bind a charge proportional to the flux. In the case of $n$ vortices, and thus $n$ flux quanta at the interface of a TI and a trivial insulator, the Josephson vortices have a charge of $Q=n \frac{e}{4}$ at the interface. The vortices in JJAs are spatial arrangements of the phase of the superconducting order parameter, which move through the array in response to forces generated. For example, Josephson vortices move perpendicularly to the external current $[19,20]$. We can make use of the Aharonov-Bohm type of interference experiment to observe the fractional charges. The effect results in charged particles acquiring a phase, $\Delta \phi=\frac{q}{\hbar} B \cdot S$, when moving around an external magnetic field. Here $B$ is the external magnetic field and $S$ is the area where the magnetic field is present. If there is a ring-like geometry, the phase picked up by the moving Josephson vortices leads to oscillation of the $I_{c}(B)$ of the JJA (Illustrated in figure 1). The period of this oscillation depends on the geometry of the JJA and the size of the hole. In addition to the conventional charge (in form of Cooper pairs), if the fractional

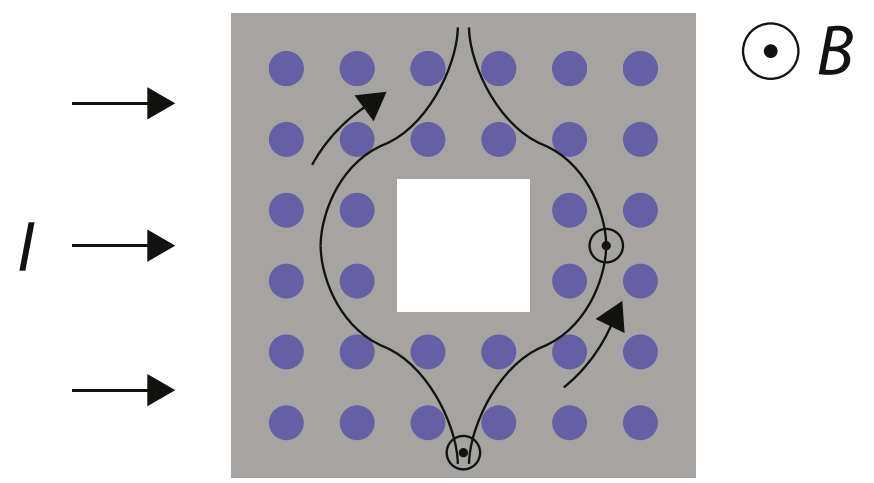

Figure 1. Interference of Josephson vortices in a JJA. An illustration drawing of interference of moving Josephson vortices (black circles) in a JJA (blue islands) with an hole in the middle. The $e / 4$ fractional charges will lead to an 4 times larger period.

charges are present in a JJA, the phase picked up by the $e / 4$ charge is 4 times smaller, resulting in a $8 \pi$-periodic oscillation. It is this configuration that was proposed by Nogueira et al [9] to detect the fractional charge formed due to the axion dynamics.

Here we report the experimental results of a TI-based JJA with proposed configuration, and of a Au-based JJA as a nontopological reference. The TI used for this experiment is $\mathrm{BiSbTeSe}_{2}$ (BSTS) with gapped bulk states [21]. In the Aubased devices only an oscillation due to the Cooper pairs is expected, while oscillations of $I_{c}(B)$ due to both Cooper pairs and the fractional charge are expected to be observed for the BSTS devices. The results are fitted with numerical simulations.

\section{Fabrication and devices}

The Au and BSTS devices were fabricated in similar ways. BSTS (1112) is a Bi-based TI with quintuple-layer structure and the Dirac point of the surface state lies in the bulk gap. Niobium is a commonly used s-wave superconductor. Both materials have been used for making superconducting devices in the our previous work [21]. For the BSTS device, high quality BSTS single crystals were grown using a modified Bridgman method [21]. BSTS crystal flakes were mechanically exfoliated on $\mathrm{SiO}_{2} / \mathrm{Si}^{++}$substrates. The JJA and electrodes were defined by standard e-beam lithography (EBL). We deposited a layer of $60 \mathrm{~nm} \mathrm{Nb}$ by DC-sputtering, which is followed by a $2 \mathrm{~nm}$ capping layer of Pd. A subsequent EBL step is used to define the hole, which is then etched by $\mathrm{Ar}^{+}$ milling. The individual islands of the JJA are $150 \mathrm{~nm}$ in diameter and are spaced $250 \mathrm{~nm}$ apart. A scanning electron microscope (SEM) image of the device is shown in figure 3(a).

For the fabrication of the Au device, a rectangle of $6 \mu \mathrm{m} \times 5 \mu \mathrm{m}$ with a square hole of $2.5 \mu \mathrm{m} \times 2.5 \mu \mathrm{m}$ is patterned using EBL. We deposited a layer of $2 \mathrm{~nm}$ Ti and $40 \mathrm{~nm} \mathrm{Au}$ by sputter deposition. Before lift-off, the device is etched by an $\mathrm{Ar}^{+}$milling under a small angle while rotating the sample. This is to remove Au walls at the edges which are 
(a)

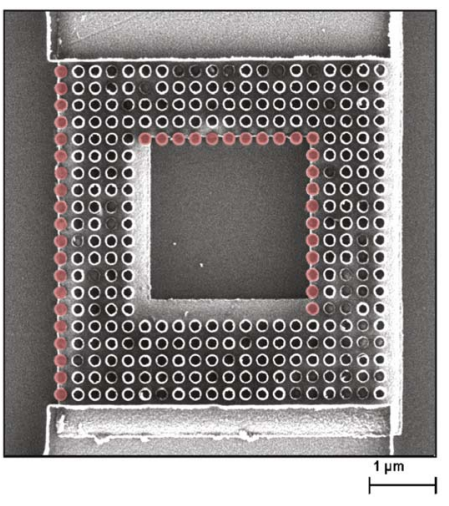

(b)

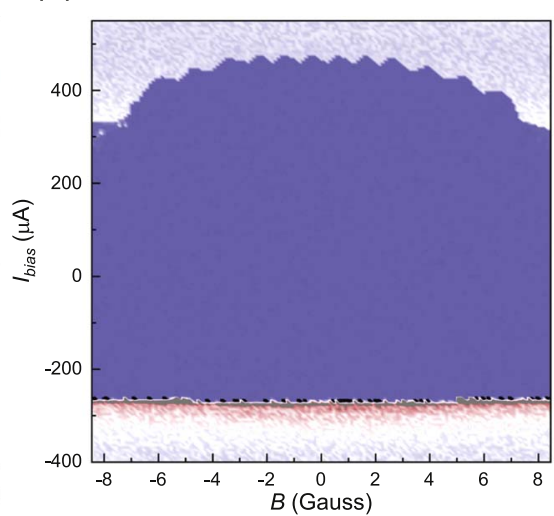

(c)

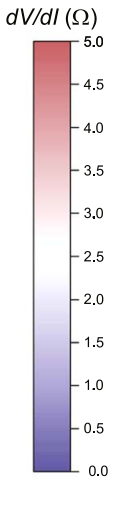

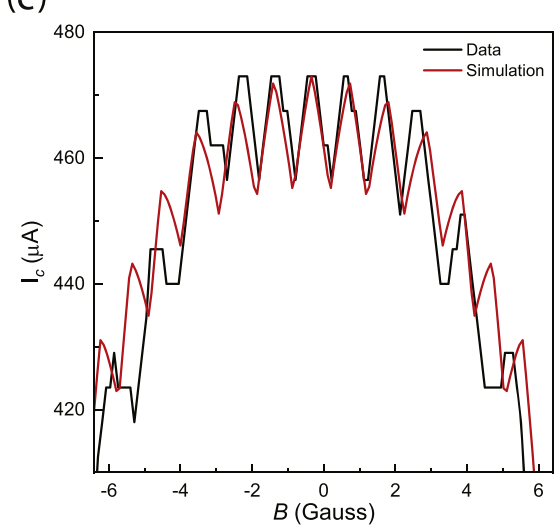

Figure 2. Au device. (a) A HR-SEM image of the Au device of 20 by $20 \mathrm{Nb}$ islands with a hole of 10 by 10 islands. The islands are $150 \mathrm{~nm}$ in diameter and there is $250 \mathrm{~nm}$ in between the centres of the islands. Due to the misalignment of the EBL process, 3 lines are removed from the simulation model (marked with red). (b) The differential resistance of the device shown in (a) as a function of the magnetic field $B$, applied perpendicular to the array, at $T=1 \mathrm{~K}$. The bias current is swept from negative to positive bias current. The scale has been adjusted to clarify the features; gray indicates datapoints above the scale. (c) $I_{c}(B)$ deduced from the measurement depicted in (b) and the simulated $I_{c}(B)$ of the geometry shown in (a).

formed due to the lift-off process. A subsequent EBL step patterns the electrodes and the JJA islands, which again are $150 \mathrm{~nm}$ in diameter and are spaced $250 \mathrm{~nm}$ apart. We sputter deposit $60 \mathrm{~nm} \mathrm{Nb}$ and $2 \mathrm{~nm} \mathrm{~Pb}$ on this device as well. A SEM image of the device is shown in figure 2(a).

\section{Results}

\subsection{Interference measurement results_-Au device}

Figure 2(b) shows the results of measurements on the Au device. The differential resistance $(d V / d I)$ as a function of the bias current was measured at different magnetic fields. The bias current was swept from negative to positive. The measurement results where the bias was swept from positive to negative are shown in the supplementary information available online at stacks.iop.org/NANO/32/435001/mmedia. As a result of the large critical current at base temperature of the dilution refrigerator, these measurements were performed at $1 \mathrm{~K}$. The oscillation of $I_{c}(B)$ is clearly visible, which is extracted and plotted as the black line in figure 2(c). This oscillation is due to the Cooper pairs moving around the hole, after which they interfere. Simulations were performed on a JJA with the same device geometry (see supplementary information). For details on the simulations, see the methods section. The results of the simulated $I_{c}(B)$ are shown in red in figure 2(c). The simulations yield results in dimensionless units. The maximum critical current of the central lobe in the measurements is used as normalization value for the current. To convert the dimensionless field to physical values, we use $B=\eta f \cdot \phi_{0} / A_{\text {hole }}$ for the external field, where $f$ is the frustration factor, $A_{\text {hole }}$ is the area of the hole and $\eta$ is an additional prefactor which is required for fitting the experimental results. For the Au arrays, $\eta=0.54$. This prefactor is an indication of non-perfect geometry or defects in the samples. Therefore to maintain one quantized fluxoid, a large effective area is needed at low field. The effective area of one Josephson vortex is defined as $A^{*}=A_{\text {hole }} / \eta$. We will discuss this later in the discussion section. Measurements over a larger range of magnetic fields, showing clear modulations of $I_{c}(B)$ by the plaquettes, are shown in the supplementary information. These measurements verify the good quality of the JJA.

\subsection{Interference measurement results-BSTS device}

The results of the BSTS device are shown in figure 3(b). Here, measurements were conducted at $15 \mathrm{mK}$. Normal $I V$ measurements, at base temperature and at elevated temperatures, can be found in the supplementary information. For the interference measurements, the bias current was applied from the bottom to the top of the device. Clear oscillations were observed over a range of \pm 8 Gauss. The extracted $I_{c}(B)$ is shown as the black line in figure 3(d). For the simulations, we reproduced the exact geometry of the device, as shown in figure 3(c). Here, the color of the dots represent the phase of each island. The blue arrows indicate the direction and magnitude (normalized to the $I_{c}$ of each junction) of the current. The resulting $I_{c}(B)$ of the simulations is shown as the red line in figure $3(\mathrm{~d})$, where the scaling factor $\eta=0.50$. The results of the measurements and the simulations are in great correspondence as the asymmetry and the shape of the envelope match. However, the simulations do not take topological effects into account. In addition to the measurements at small magnetic fields, we also measured the device for larger magnetic fields (supplementary information), where again a clear modulation in $I_{c}(B)$ at a period belonging to $A_{\text {plaquette }}$ is present, once more indicating a high quality JJA.

\section{Discussion}

In conclusion, both the Au and BSTS devices show an oscillation of the $I_{c}(B)$ as a result of the Cooper pairs moving 
(a)

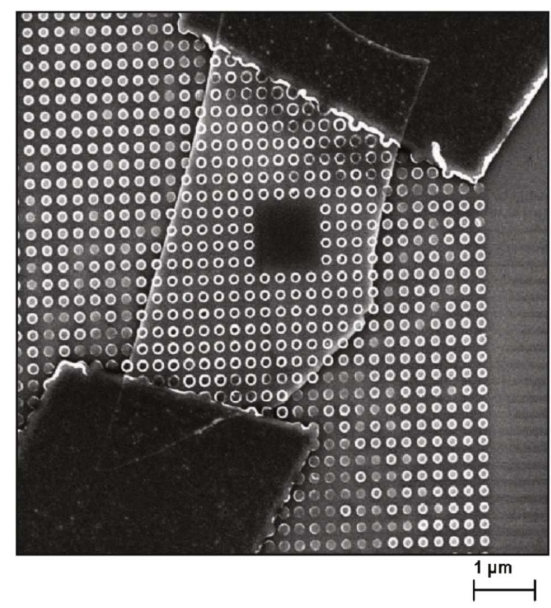

(b)

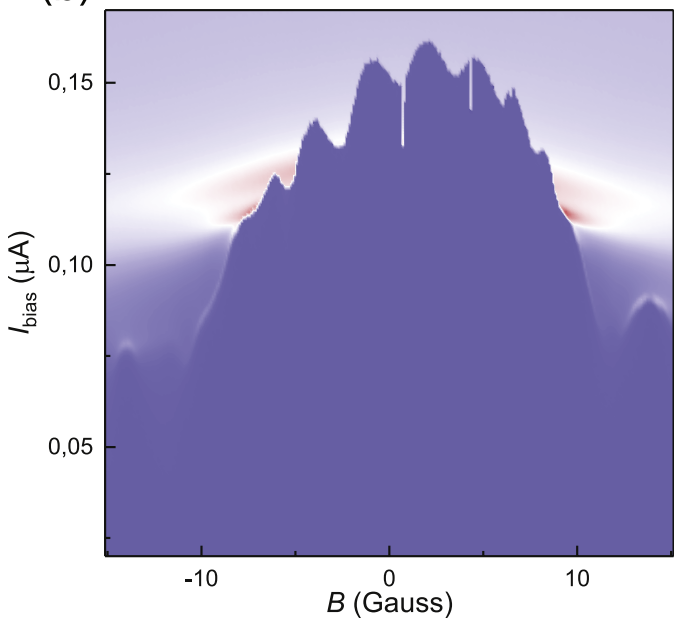

$d V / d l(\mathrm{k} \Omega)$

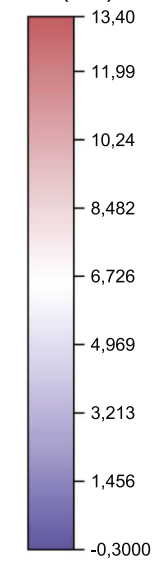

(c)

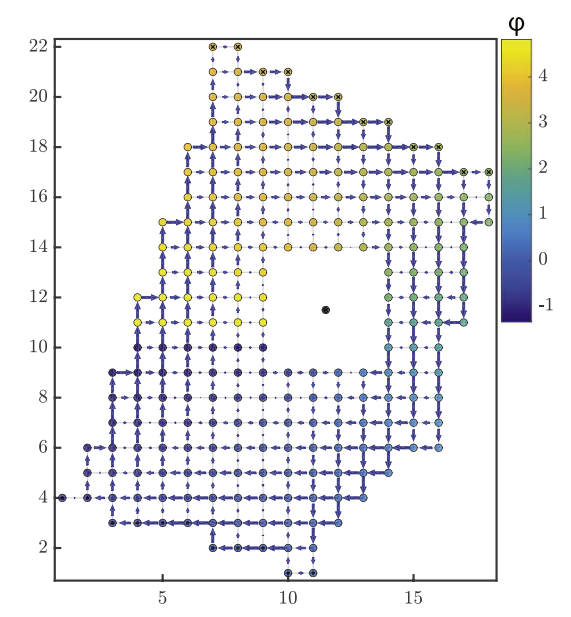

(d)

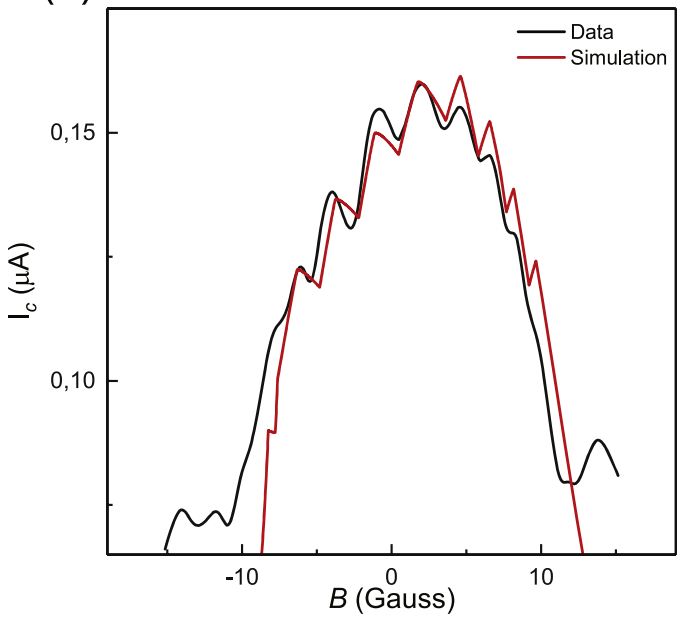

Figure 3. BSTS device. (a) A HR-SEM image of the BSTS device with a hole of 4 by 4 islands. The islands are $150 \mathrm{~nm}$ in diameter and there is $250 \mathrm{~nm}$ in between the centres of the islands. (b) The differential resistance of the device shown in a as a function of the magnetic field $B$, applied perpendicular to the array, at $T=15 \mathrm{mK}$. (c) The geometry used to simulate the $I_{c}(B)$ of the device shown in (a). (d) $I_{c}(B)$ deduced from the measurement depicted in (b) and the simulated $I_{c}(B)$ of the geometry shown in (c).

around the perpendicular magnetic field inside the hole. This is also verified by the performed simulations. The expected oscillation period in magnetic field $\left(\Delta B=\Phi_{0} / A\right)$ is related to both the area of the hole $\left(A_{\text {hole }}\right)$ and the total area of the arrays $\left(A_{\text {total }}\right)$ and therefore lies between $\Phi_{0} / A_{\text {total }}$ and $\Phi_{0} / A_{\text {hole }}$. For the Au sample, having a lattice constant $a=250 \mathrm{~nm}$ and being a $20 \times 20$ array with a $10 \times 10$ hole, we find $\Delta B_{\text {total }}=0.83$ Gauss and $\Delta B_{\text {hole }}=2.30$ Gauss, where for the latter we take the area of the hole spanned by the first ring of islands, being a $12 \times 12$ ring. The measured effective area, $A^{*}$, of approximately 1 Gauss is then exactly $A_{\text {hole }} / \eta$. Note that the value of $\eta$ is computed by finding an optimal scaling factor between the measurement data and simulation data by means of a fit procedure, and hence does not take any explicit area into account. In addition to the relatively large areas of the hole and array, we also consider the array of the plaquette, where $A_{\text {plaquette }}=a^{2}$ so that $\Delta B_{\text {plaquette }}=33.1 \mathrm{mT}$. A scan of $d V / d I$ as a function of bias current over a wide magnetic field range is shown in the supplementary information, where resonances at (fractional) frustration factors can be observed. This is in general an indication of the high quality of the superconducting arrays.

\subsection{Prefactor and effective area}

The discrepancy between the experimental results and the simulation lie in the fabrication-induced artificial defects which can be described by the prefactor $\eta$ or an effective area $A^{*}=A_{\text {hole }} / \eta$. These defects can be induced due to bad liftoff process or misalignment of the EBL patterning. For example, in the $\mathrm{Au}$ sample, the misalignment possibly resulted in missing lines of arrays or reduced $I_{c}$ (figure 2(a)). Therefore, the effective area of the hole can be larger than that of the design. A comparison of the simulations with and without geometry corrections for the Au device is shown in the supplementary information (section 5). With the same fitting procedure, the simulation with geometry correction matches better the experimental result, yielding a larger 


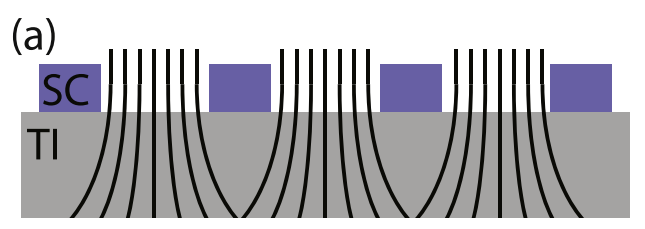

(b)

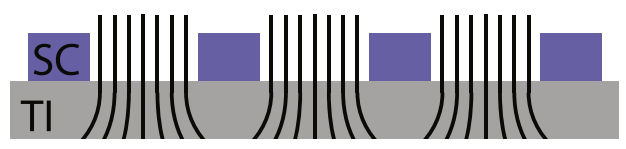

Figure 4. Magnetic field through flakes of different thicknesses. (a) Schematic side view of a JJA on a relatively thick TI flake. Here the flux becomes homogeneous the bottom surface. (b) Schematic side view of a JJA on a relatively thin TI flake. Here the flux does not overlap at the bottom surface.

prefactor (0.54), closer to 1 . Also, the amplitude is larger, which is closer to the experimental results. And some of the peaks are shifted, getting closer to the experimental results. Additionally, the patterns were written without proximity effect correction. Therefore, islands in the middle of the array are slightly overexposed, making them wider and hence increasing their local $I_{c}$. This would also effectively increase the effective area. However, the exact fitting (prefactor $=1$ ) needs the current density distribution which is hard to define or measure accurately.

A similar situation can be found in the TI-based device (figure 3(a)), in which the simulation captured every single junction according to the SEM image, but the irregular shape of the flake and the inhomogeneity in the critical current distribution (partially sitting on the edge, bad lift-off close to the leads) resulted in a larger effective area than the simulation.

To further improve the signal, highly efficient and highyielding patterning of the arrays is desired.

\subsection{Topological effect}

No clear effects of the topological fractional charge in the BSTS device was observed. A reason for this may be that the charge density induced by the magnetic vortices is small compared to the charges that contribute to the current. Secondly, the opposite effect of the other surface of the BSTS flake may cancel the total effect. If the flake is too thin the focused flux will be present at both surfaces (figure 4(b)). As $\Delta \theta$ has opposite signs at both surfaces of the BSTS flake, the effect may be canceled. However, if the flake is thick enough, the flux may spread out and become homogeneous at the bottom surface.Then the effects of the fractional charge will be preserved. A schematic draw is shown in figure 4. Based on the study of superconducting vortices $[22,23]$ the depth of focused flux is estimated to be about $500 \mathrm{~nm}$ (see supplementary information for more details). The typical thickness of the BSTS film is about $100 \mathrm{~nm}$. In addition, the oscillation of the $I_{c}(B)$ due to the topological fractional charge is $8 \pi$ periodic, while only 7 clear peaks of the $I_{c}(B)$ are measured. By increasing the island density and reducing the distance between the islands, the $I_{c}$ is expected to increase, while the $I_{c}(B)$ is expected to decrease at a smaller rate. This is expected to increase the number of measured peaks of $I_{c}(B)$ in order to measure signatures of the fractional charge as a result of topological effects. It is worth noticing that for the BSTS sample, there is a background modulation of the $I_{c}$ besides the central lobe, resulting in a small increase of $I_{c}$ comparing to the simulation result (figure 3(d)). This modulation effect can be seen more clearly in a larger range data set (supplementary information, section D). These lobes are roughly half the size of the middle lobe, similar to the Fraunhofer pattern-like feature and coincidentally about 4 times the period of holerelated oscillation. It needs further investigation to verify whether this is due to the geometry effect or topological properties.

\section{Methods}

To verify the measurements, simulations on JJA were performed by the simulation package JJAsim developed by $\mathrm{M}$ Lankhorst [24]. These simulations are based on the resistive shunted capacitive junction model. The array is modeled by $j$ single junctions, for which

$$
\begin{gathered}
I_{j}=I_{c_{j}} \sin \left(\varphi_{j}\right)+\frac{\hbar}{2 e R_{j}} \frac{\partial \varphi_{j}}{\partial t}+\frac{\hbar C_{j}}{2 e} \frac{\partial^{2} \varphi_{j}}{\partial^{2} t}+\eta_{j}(T) \\
V_{j}=\frac{\hbar}{2 e} \frac{\partial \varphi_{j}}{\partial t}+\sum_{j^{\prime} \in J} L_{j, j^{\prime}} \frac{\partial I_{j^{\prime}}}{\partial t} .
\end{gathered}
$$

Here, $I_{j}$ is the current through junction $j, I_{c_{j}}$ the critical current of junction $j, \varphi_{j}$ the phase difference of the junction, $R_{j}$ the resistance of the junction, $C_{j}$ represents the capacitance of junction $j$ and $\eta_{j}(T)$ is the temperature dependent noise realization. In equation (3), $L_{j, j^{\prime}}$ is the inductance matrix with self-inductance on the diagonal and mutual inductance off the diagonal. The dynamics of a JJA of $j$ junctions, are described by the Kirchhoff's current law, equation (4), and a Kirchhoff's law of voltage equation (5). The voltage is rewritten as gauge-invariant phase differences, where the integration constant of the time-integral does not necessary vanish.

$$
\begin{gathered}
\sum_{\substack{n \in N \\
j \in J}} M_{n, j} I_{j}+I_{n}^{\mathrm{ext}}=0 \\
\sum_{j \in P} A_{P, j} \varphi_{j}=2 \pi\left(z_{P}-\frac{\phi_{P}}{\phi_{0}}\right) .
\end{gathered}
$$

Here we denote $J$ as the set of junctions in the array and $N$ as the set of superconducting islands in the array. $M_{n, j}$ describes the current law through every island, such that $M_{n, j} I_{j}$ describes the current through island $n$ from junction $j . I_{n}^{\text {ext }}$ represents the external bias current applied to island $n$. Equation (5) describes 
the phase winding rule, as it sums over the gauge invariant phase differences along a closed path, $P$. Here, $A_{P, j}=1$, if junction $j$ is traversed by path $P$ and 0 otherwise. As a phase change of $2 \pi$ to any junction does not change the physical solution, the Kirchhoff's law of current and voltage must be invariant. The Kirchhoff's law of current is $2 \pi$-periodic. The phase zone, $z_{P}$, is introduced to make the Kirchhoff's law of voltage $2 \pi$-periodic. The phase zone, $z_{P}$ is a set of arbitrary integers, which allows to solve another instance of $\varphi_{j}$. Lastly, $\phi_{P}$ is the flux through the closed path, $P$ and $\phi_{0}$ is a flux quantum. Note that the topological effects are not taken into account for any of the simulations performed. All quantities in this simulation package are dimensionless. An overview of the normalization of the quantities is attached in the supplementary information in table S1.

\section{Data availability statement}

The data generated and/or analysed during the current study are not publicly available for legal/ethical reasons but are available from the corresponding author on reasonable request.

\section{ORCID iDs}

J M Brevoord (iD https://orcid.org/0000-0002-8801-9616

D H Wielens (ib https://orcid.org/0000-0002-2816-9671

M Lankhorst (10) https://orcid.org/0000-0002-4018-4591

C Li (ib https://orcid.org/0000-0001-5713-2117

\section{References}

[1] Fu L and Kane C L 2007 Phys. Rev. B 76045302

[2] Hsieh D, Qian D, Wray L, Xia Y, Hor Y S, Cava R J and Hasan M Z 2008 Nature 452970
[3] Chen Y L et al 2009 Science 325 178-81

[4] Ren Z, Taskin A A, Sasaki S, Segawa K and Ando Y 2011 Phys. Rev. B 84165311

[5] Fu L and Kane C L 2008 Phys. Rev. Lett. 100096407

[6] Wiedenmann J et al 2016 Nat. Commun. 710303

[7] Sun H-H et al 2016 Phys. Rev. Lett. 116257003

[8] Schüffelgen P et al 2019 Selective area growth and stencil lithography for in situ fabricated quantum devices Nat. Nanotechnol. 14 825-31

[9] Nogueira F S, Nussinov Z and Van den Brink J 2018 Phys. Rev. Lett. 22121

[10] Qi X-L, Hughes T L and Zhang S-C 2008 Phys. Rev. B 78 195424

[11] Qi X-L, Li R, Zang J and Zhang S-C 2009 Science 323 5918

[12] Li Q, Kharzeev D E, Zhang C, Huang Y, Pletikosić I, Fedorov A V, Zhong R D, Schneeloch J A, Gu G D and Valla T 2016 Nat. Phys. 12 550-4

[13] Zhang C et al 2017 Nat. Commun. 813741

[14] de Boer J C, Wielens D H, Voerman J A, de Ronde B, Huang Y, Golden M S, Li C and Brinkman A 2019 Phys. Rev. B 99085124

[15] Witten E 1979 Phys. Lett. B 86 3-4

[16] Peterson C W, Li T, Jiang W, Hughes T L and Bahl G 2021 Nature $\mathbf{5 8 9} 376$

[17] Li T, Zhu P, Benalcazar W A and Hughes T L 2020 Phys. Rev. B 101115115

[18] Peterson C W, Li T, Benalcazar W A, Hughes T L and Bahl G 2020 Science 3681114

[19] Wenbin Y, Lee K H and Stroud D 2003 Phys. Rev. B 47 5906

[20] Rzchowski M S, Benz S P, Tinkham M and Lobb C J 1990 Phys. Rev. B 422041

[21] de Ronde B, Li C, Huang Y and Brinkman A 2020 Nanomaterials $\mathbf{1 0} 4$

[22] Chang A M, Hallen H D, Harriott L, Hess H F, Kao H L, Kwo J, Miller R E, Wolfe R and van der Ziel J 1992 Appl. Phys. Lett. 611974

[23] Brisbois J, Raes B, de Vondel J V, Moshchalkov V V and Silhanek A V 2014 J. Appl. Phys. 115103906

[24] Lankhorst M JJAsim: Josephson circuit simulator for arrays (http://jjasim.com/) 